REVISTA ANDALUZA DE ANTROPOLOGÍA

NÚMERO 17: ESTRATEGIAS SUBALTERNAS EN AMÉRICA LATINA: RECONFIGURANDO LA IDENTIDAD PARA ARTICULARSE A UN MUNDO GLOBAL. SUBALTERN STRATEGIES IN LATIN AMERICA: RECONFIGURING IDENTITY TO BE ARTICULATED TO THE GLOBAL WORLD.

SEPTIEMBRE DE 2019

ISSN 2174-6796

[pp. 115-136]

http://dx.doi.org/10.12795/RAA.2019.17.06

\title{
ANÁLISIS DE LA CONFLICTIVIDAD SURGIDA POR LA VISIBILIZACIÓN DE POBLACIÓN DROGODEPENDIENTE, EN ESPACIOS DE MADRID CAPITAL
}

\section{ANALYSIS OF THE CONFLICT ARISING FROM THE VISIBILITY OF DRUG-DEPENDENT POPULATION, IN SPACES OF MADRID CAPITAL}

\author{
Diego Fernández Piedra \\ Doctor en Sociología y Antropología Social \\ Ayuntamiento del Real Sitio de San Ildefonso (Madrid)
}

\section{Resumen.}

Este texto, después de una explicación sobre el "problema de la droga" y la conflictividad surgida de este paradigma, explica los usos que ciertos colectivos de drogodependientes hacen de algunos espacios públicos de la ciudad de Madrid, para posteriormente reflexionar sobre las problemáticas producidas en ellos. Esto no puede ser comprendido desde un planteamiento simplista, con lo que el análisis de los actores que participan en ellas, resulta clave para su comprensión. Con este fin tendremos en cuenta no sólo a la población drogodependiente, sino el papel que desempeñan los dispositivos asistenciales, vecinos y comerciantes de las zonas. En la parte final del artículo, planteamos la necesidad de generar nuevos modelos de intervención, más acorde a la realidad actual, sustentados en la participación de todos agentes los implicados.

Palabras clave.

Políticas sobre drogas; Heroína; Conflictividad Urbana; Nimby. 


\begin{abstract}
.
This text, after an explanation about the "drug problem" and the conflict arising from this paradigm, explains the uses that certain groups of drug addicts make of some public spaces of the city of Madrid, to later reflect on the problems produced in they. This can not be understood from a simplistic approach, so that the analysis of the actors involved in them is key to understanding them. To this end, we will take into account not only the drug-dependent population, but also the role played by assistive devices, neighbors and merchants in the zones. In the final part of the article, we propose the need to generate new models of intervention, more in line with current reality, based on the participation of all those agents involved.
\end{abstract}

\title{
Key Words.
}

Drug policies; Heroin; Urban Conflictivity; Nimby.

\section{Introducción.}

El siguiente artículo nace del análisis del material obtenido de una investigación encargada por el Instituto de Adicciones de Madrid Salud. Esta institución contrató a la Asociación Proyecto Hogar (entidad especializada en mediar en problemáticas del ámbito de las drogodependencias) para realizar un estudio sobre la "Situación de la problemática de las adicciones en el Puente de Vallecas”, ver en Fernández Piedra y Corral (2018).

Si bien el anterior trabajo no tuvo como objetivo analizar el proceso que lleva a definir como "problema" un espacio en el que interactúan drogodependientes y otras poblaciones, logramos de manera transversal suficiente información para poder descifrar esta conflictivización del lugar y de las representaciones sociales referidas a la inseguridad. Así como las prácticas y tomas de decisiones articuladas en ellos, para conseguir reconducir la situación al orden social aceptado como correcto localmente.

\section{El “problema de la droga”.}

Megías et al. $(2000)^{2}$ y Romaní $(1999)^{3},(2010)^{4}$ utilizan este concepto para referirse a un proceso acontecido en España en las décadas 70 y 80 del siglo XX. Durante este período,

1. Fernández Piedra, D. y Corral, G. (2018). “Situación de la problemática de las adicciones en el Puente de Vallecas”. Referencia de Exp.180201702546. Madrid. Madrid Salud Instituto de Adicciones.

2. Megías, E., et al. (2000). “La percepción social de los problemas de drogas en España”. Madrid. FAD.

3. Romaní, O. (1999). “Las Drogas. Sueños y razones”. Barcelona. Ariel.

4. (2010). "Adicciones, drogodependencias y "problema de la droga" en España: la construcción de un problema social. Cuicuilco. 17. Pp (83-101). 
coincidiendo con la transición, se generó un referente explicativo de las adicciones. El cual negativizó aquello relacionado con estas, modificando su percepción. Esta representación emergió de extrapolar casos en los que usuarios de heroína desarrollaron una adicción marcada por el deterioro físico, configurando un arquetipo sustentado en la peligrosidad de estos consumos para la salud.

Autores como Pallarés $(1996)^{5}$, Comas $(1989)^{6}$, Escohotado $(1996)^{7}$, Gamella $(1997)^{8}$, etc. aseveran que, entender la "droga como problema", produce paradigmas interpretativos, discursos, percepciones, modos de intervención, interacciones con la población no consumidora, etc. en términos de peligro, marginalidad, criminalización y evitación. Generando una necesidad interventiva en términos de alarma social. La cual aumenta, según nuestras observaciones, exponencialmente según la cantidad de consumidores identificados en los diferentes espacios públicos de Madrid capital.

Distintas respuestas sustentadas en esta demonización de la droga, tanto institucionales como de la sociedad civil, se han implementado en los últimos años. Pero como defienden Sepúlveda, Báez y Montenegro (2008) ${ }^{9}$, a pesar de ellas, el consumo de casi todas las drogas se ha elevado al estar fundamentadas dichas intervenciones en verdades sesgadas.

Actualmente se atisba un cambio en las percepciones manejadas sobre esta problemática. Si bien el anterior estigma todavía está presente al definir las adicciones, convive con interpretaciones más reales. Normalizando situaciones antes impensables. No sólo el consumo de tabaco, alcohol y cannabis es algo que no genera alarma en nuestra sociedad, sino que ciertos usos recreativos de cocaína, drogas de síntesis, etc. tampoco lo hacen, como resaltan Martínez y Pallarés (2013) ${ }^{10}$.

Vallecas es un barrio donde el consumo de heroína produjo mayor cantidad de pérdidas a nivel económico, físico, social, etc en todo Madrid, al igual que una normalización de 5. Pallarés, J. (1996). “El placer del escorpión. Antropología de las heroína y los yonquis”. Lérida. Milenio.

6. Comas Arnau, D. (1989). "La construcción social de la imagen del drogodependiente: consecuencias de la prevención y la atención”. Madrid. Inserso.

7. Escohotado, A. (1986). "La creación del problema (1900-1929)". En "Revista española de investigaciones sociológicas”, 34, pp (3-56). Madrid.

8. Gamella, J. (1997). "Heroína en España, 1977-1996. Balance de una crisis de drogas". En Claves de Razón Práctica, 72, pp (20-30). Madrid.

9. Sepúlveda, M., Báez, F. Y Montenegro, M. (2008). "No en la puerta de mi casa. Implantación no conflictiva de dispositivos de drogodependencias”. Barcelona. Grup Igia.

10. Martínez Oró, D y Pallarés Gómez, J. (2013). "Riesgos, Daños y Placeres: A modo de introducción de un manual para entender las drogas". En "De riesgos y placeres: Manual para entender las drogas". P.p (23-36). 
estas. Produciéndose esto, más por un desconocimiento sobre la sustancia, que por ella misma. Este período es definido por su vecindario en términos de "epidemia", concepción sustentada en "el problema de la droga". Por eso en Vallecas encontramos conviviendo discursos y reacciones muy duras sobre las drogas, junto a otros más suaves cercanos a la aceptación del consumo.

\section{Metodología.}

Para la consecución de los objetivos propuestos para la investigación que sustenta este texto, planteamos un diseño cualitativo centrado en el método etnográfico. Junto a este, realizamos un análisis de datos estadísticos relacionados con el fenómeno, complementándolo con una revisión bibliográfica sobre el objeto de estudio.

Durante los 5 meses que duró este trabajo, acudimos de manera regular a espacios de permanencia de población drogodependiente. Asistiendo primero a los sugeridos por Proyecto Hogar, para posteriormente centrarnos en aquellos que surgieron de la propia investigación.

La principal técnica de producción de datos utilizada, fue la observación participante. A la par y con similar importancia, utilizamos la entrevista semiestructurada. Estas fueron realizadas a población drogodependiente, comerciantes y vecindario de las zonas observadas. La selección de los participantes siguió un criterio de muestreo teórico y de opinión, Glaser y Strauss $(1967)^{11}$. No buscando una representatividad estadística, sino captar los diferentes factores que influyen en la configuración de la opinión.

Todos aquellos individuos entrevistados y con los que mantuvimos conversaciones informales, supieron en todo momento el uso que se haría de sus datos. Comenzando cualquier interacción con el uso de un consentimiento informado.

Las instituciones contratantes conocieron y aprobaron la utilización delos datos recabados, para generar todo tipo de publicaciones. Siempre manteniéndose el anonimato de los informantes.

\section{La heroína en España y el caso de Madrid.}

El fenómeno de las adicciones, solo puede entenderse a través de las transformaciones económicas, sociales y culturales acontecidas durante la Revolución Industrial, como explican Berger y Kellner $(1985)^{12}$. Siendo el desarrollo del modo de producción

11. Glaser, B. \& Strauss, A. (1967). “The discovery of grounded theory”. Chicago. Aldine Press.

12. Berger, P y Kellner, H. (1985). "La reinterpretación de la sociología”. Madrid. Espasa Calpe. 
capitalista y los cambios acontecidos durante la "modernización" (movilidad, división social, etc.) centrales para su comprensión.

Estas modificaciones provocaron, entre otros fenómenos, una descontextualización de usos y sustancias. Aquello, anteriormente utilizado con un fin y en un entorno ritual/ biomédico, se deslocalizó. Las normas y pautas de consumo, construidas para evitar su uso fuera de sus ambientes primigenios, perdieron su utilidad inicial. Modificando el sentido que tenían las drogas hasta entonces.

Generando este proceso la aparición de nuevos elementos reguladores, acordes con las formas de gobierno actuales. Concretándose en lo que Rose, O 'malley y Valverde (2006) ${ }^{(13)}$, llamaron "gobierno en la distancia". El cual se caracteriza por articular dispositivos que aleccionan a la población en métodos para "autogobernarse", cumpliendo los preceptos previamente pautados por dichas instituciones.

En el campo de las drogas, se articularon dispositivos disciplinantes sobre aquello considerado como saludable. Sirviendo estos como respuesta a las nuevas sustancias, contextos y usos que aparecieron derivados de la industrialización. Recursos que, entre otros cambios sustanciales, responsabilizaron al usuario de drogas de su consumo y recuperación, proceso del cual ellos eran los responsables.

En España la autarquía que caracterizó la dictadura franquista, propició que la problemática de las drogas apareciese más tarde que en resto de Europa. Determinando el inicio de la transición democrática el desarrollo del fenómeno de las adicciones.

Romaní (1999) ${ }^{13}$, explica cómo durante la década de los '70, España contaba con numerosos consumidores de opiáceos y hachís relacionados con lo bohemio y marginal. Este tipo de usos, si bien eran reprobados por el resto de la sociedad, no fueron definidos socialmente como problemáticos. La heroína, vinculada a elementos contraculturales, no apareció en España hasta 1973. Considerándose durante varios años una droga más. Relacionando su consumo a individuos progresistas y no a lo marginal.

En 1977 las variaciones políticas modificaron la realidad de las adicciones. Plasmándose estas en las pautas de consumo de alcohol, donde se introdujeron los combinados y la cerveza.

Por su parte, el tabaco se normalizó, llegando a él las mujeres. Además, sustancias como el hachís, pierden su carga bohemia. Aumentando su consumo a la par que su estigma.

13. Rose, N., O` malley, P. y Valverde, M. (2006). “Governmentality”. Annual Review of Law \& Society, 2, pp (83-104). Barcelona. 
Coincidiendo con el triunfo socialista, la heroína llegó a individuos provenientes de grupos marginales. Asumiendo los ya existentes mercados fuera de la ley, el negocio de esta sustancia. Proceso que propició la pérdida del carácter elitista que siempre la había acompañado, consolidándose así la relación entre marginal-droga.

Respecto a Madrid, podemos destacar que sufrió un proceso similar, imbricándose las drogas en un barrio como Vallecas por los motivos que a continuación vamos a resaltar.

Este municipio, en sus orígenes, fue un municipio independiente de la ciudad de Madrid, anexionado en el año 1950. Conformado en sus inicios por inmigrantes españoles, esta población se asentó en los arrabales de la ciudad, al no poder residir en la capital por la falta de espacio y capacidad económica. Construyendo viviendas de poca calidad, denominadas "chabolas". Con el paso del tiempo y tras varias decisiones institucionales, estas personas las abandonaron siendo realojados en otros espacios. Provocando que estos lugares desechados, fueran ocupados por población de etnia gitana, mayoritariamente.

La creciente demanda de heroína (coincidiendo con el ocaso de la dictadura) encontró en este barrio y en algunos de sus residentes, el caldo de cultivo perfecto para establecer su compra/venta, al contar con estructuras de mercado fuera de la ley. Estas, pudieron dar respuesta a la demanda existente en toda la capital. Situación que, junto a la falta de información y la lentitud de respuesta institucional, propició que este distrito madrileño se convirtiera en uno de los grandes referentes respecto a esta sustancia del imaginario social español.

La aparición del SIDA, asociado al consumo de drogas por vía parenteral, maximizó la negativización vinculada a estas. Legitimando un cambio en la intervención.

\section{Los dispositivos Asistenciales.}

Santodomingo (2009) ${ }^{14}$ y Fernández Piedra $(2018)^{15}$, señalan cómo en Europa desde 1972 se comenzaron a utilizar los "programas libres de drogas". Su intervención se centraba en la obtención de la abstinencia sin utilizar fármacos. Olvidando otros factores que también influyen en este fenómeno.

14. Santodomingo, J. (2009). "Historia de las adicciones y su abordaje en España". En "Historia de las adicciones en la España contemporánea”. pp (13-36). Madrid. Ministerio de Sanidad y Consumo.

15. Fernández Piedra, D. (2018). "Narcóticos Anónimos y Centros de Atención al Drogodependiente: un análisis antropológico de dos recursos asistenciales". (Tesis doctoral). Madrid. Universidad Complutense de Madrid. 
Estos tratamientos, junto a políticas centradas en la prevención y penalización/ criminalización de las adicciones, provocaron una disminución e invisibilización del consumo, pero no su erradicación.

La posterior irrupción de paradigmas que interpretaban el fenómeno con mayor complejidad propició la aparición de recursos plurales. Sustentados en la expansión de lo social, al abrigo del Estado del Bienestar. Dando paso a los programas de "reducción de daños", los cuales buscan disminuir las consecuencias negativas del consumo de drogas.

Entre 1975 y 1979, aparecieron las "Comunidades Terapéuticas". Ofreciendo una alternativa asistencial a la hospitalaria, vinculada anteriormente con instituciones dedicadas al tratamiento de enfermedades mentales.

En 1979 se abrió el primer Centro de Atención al Drogodependiente en Madrid, ubicado en el centro de la ciudad. Conformado en su inicio por personal sanitario y ofreciendo sólo programas libres de droga. Posteriormente, se abrieron centros similares en los principales distritos del municipio.

Actualmente, esta ciudad cuenta con dos redes asistenciales de tratamiento de adicciones. Una cuya competencia corresponde a la Comunidad Autónoma y otra al Ayuntamiento. Contando ambas con dispositivos asistenciales propios, en los que se entrelazan tratamientos libres de droga, con metadona y de reducción de daños. Llevados a cabo por profesionales tanto sanitarios como de otras disciplinas.

Fuera delámbito estatal, encontramos ONG's como Proyecto Hogar, Cáritas, Alcohólicos Anónimos, etc. e instituciones privadas como Narconon, UniADIC, etc. las cuales ofrecen tratamientos similares.

Como explican Sepúlveda, Montenegro y Báez $(2007)^{16}$, cambios como el descenso de la mortalidad vinculada al consumo de heroína y la resignificación de los consumidores como "enfermos", provocados por las modificaciones interventivas que generó la irrupción de nuevos modelos explicativos sobre las drogas, alteraron tanto el imaginario social como el discurso hegemónico sobre ellas.

Actualmente, la "epidemia de heroína" funciona como referente explicativo de la problemática existente con las drogas y sus tratamientos en Vallecas y el resto de Madrid. Pese a que el mantenimiento del consumo de drogas tradicionales y la aparición de nuevas muestran cómo este abordaje en términos de problema no es sólo incorrecto, sino que sesga la realidad de este fenómeno. Mostrando este mantenimiento ideológico conflictiva de dispositivos de drogodependientes". Barcelona. Grup Igia. Colección Cuestiones emergentes. 
las múltiples contradicciones, pendientes de reflexión, existentes en el campo de las adicciones. Siendo una de las más evidentes la que presenta a los consumidores como enfermos/marginados. Los cuales tienen derecho a recibir tratamiento y protección estatal, pero a la vez conviven con un estigma tan poderoso que genera movimientos de rechazo social hacia ellos y sus tratamientos.

\section{La visibilización de población drogodependiente.}

La mercantilización de algunas de estas sustancias (tabaco, alcohol, hipnosedantes, etc.), las cuales son un producto más asociado a la salud/ocio que a la adicción; el error de teorías como "la escalada" y el "modelo evolutivo en el consumo de drogas" de Kandel $(1980)^{17}$; la normalización del consumidor/enfermo en vez del adicto/peligroso; los cambios acontecidos en los tratamientos, donde en la actualidad empiezan a tener cabida conceptos como "consumo responsable"; etc. han conseguido que parte de la interpretación sobre este fenómeno en la actualidad, ya no se realice en términos de alarma. Ocasionando un descenso notable en la percepción del riesgo asociado al fenómenos de las adicciones.

Aunque todavía podemos encontrar ciertas situaciones donde esta percepción se recrudece, articulando respuesta sustentadas en un sentimiento de alarma que perdura atávicamente en el inconsciente vallecano.

Ejemplo de la reflexión anterior lo encontramos en el fenómeno denominado "Not in my Back Yard" (NIMBY) o en su versión castellanizada "Sí pero aquí no" (SPAN). Alberdi, Peña e Ibarra. $(2002)^{18}$, Nello (2003) ${ }^{19}$, Sepúlveda, Báez y Montenegro (2008) ${ }^{20}$ y Mardones (2009) $)^{21}$ se refieren a él como "la reacción que se produce en algunos agentes sociales, los cuales se organizan con el objetivo de oponerse al riesgo percibido, ante la implementación en su contexto de ciertas actividades o instalaciones identificadas como una amenaza, sin oponerse a las mismas".

17 Kandel, D.B. (1980). "Developmental stages in adolescent drug involvement". "Theories on drug abuse". Rockville,Maryland. National Institute on Drug Abuse.

18. Alberdi, J., de la Peña, A. e Ibarra, P. (2002). “Una Reflexión Sobre La Acción Colectiva Nimby: El Caso Hontza”. En Inguruak, Revista de Sociología y Ciencia Política. 33, pp (59 - 79). Bilbao.

19. Nello, O. (2003). “¡Aquí, no!: Els conflictes territorials a Catalunya”. Barcelona. Ediciones Empuries.

20. Sepúlveda, M., F. Báez Y M. Montenegro. (2008). "No en la puerta de mi casa. Implantación no conflictiva de dispositivos de drogodependencias". Barcelona. Grupo Igia.

21. Mardones Arévalo, R. (2009). “ “No en mi patio trasero!: el caso de la comunidad ecológica de Peñalolén!” En Íconos. Revista de Ciencias Sociales. 34, pp. (139-149). Quito. 
Esta suceso, como explica Martín-Crespo $(1996)^{22}$, se desencadena ante la instalación de una depuradora, una incineradora de basuras, un aeropuerto, una antena de telefonía móvil, recursos asistenciales para drogodependientes, etc. Las primeras noticias de la implementación de estos proyectos, así como la visualización de situaciones identificadas como peligrosas por el vecindario, generan un aumento de la percepción de peligro en todo el colectivo. El cual, es instrumentalizado y utilizado como justificación para llevar a cabo intervenciones reaccionarias que minimicen esa amenaza que ellos mismos han generado.

El fenómeno NIMBY, relacionado con las adicciones y la realidad vallecana, ejemplifica la contradicción enfermo/marginado a la que nos hemos referido anteriormente. Ya que este contexto, debido a su relación histórica con las drogas, conoce los beneficios que aportan los recursos de atención a consumidores y no alarmándose ante su presencia. Pero a su vez, la construcción de estos dispositivos en su entorno inmediato y la acumulación de sus usuarios, hace que sean identificados como negativos. Articulando un proceso temporal de instrumentalización del riesgo y de movilidad en las representaciones sociales sobre las adicciones, que justifique actuaciones contrarias a su gran capacidad empática con este fenómeno. Representaciones que volverán a su posición de origen, cuando la "amenaza" no se perciba como tal.

El desencadenante de esta situación suele ser suceso escabroso, que precipita la toma de decisiones vinculadas con el proceso anteriormente descrito. Pudiendo ser desencadenarlos situaciones como la aparición de "narcopisos" (viviendas en las que se vende y consume droga), un evento de violencia extrema, una gran pelea, la existencia de "kundas" (vehículos que acercan a población drogodependiente a zonas de compraventa), etc. Esto, visibiliza un tipo de población antes "oculta”. Provocando la aparición del proceso analizado en este artículo, el cual sirve como sustento a discursos y reacciones duras por parte del vecindario, buscando su difuminación.

\section{El dispensario de Calella. Kundas. Parques, plazas y Narcopisos. Problemáticas generadoras de movilidad, en las representaciones sobre las adicciones en Madrid.}

Durante nuestro período de investigación pudimos observa varios episodios, los cuales desencadenaron reacciones por parte del vecindario como las que estamos analizando en este artículo.

\section{a) La proliferación de las kundas.}

22. Martín-Crespo, M. (1996). "Por qué sí y por qué no en mi patio de atrás. Una revisión del concepto de sindrome "Nimby" (Not In My Back Yard) en torno al tema de la gestión de residuos radiactivos". En Política y Sociedad, 23, pp (147-152). Madrid. 
Nuestro primer acercamiento a una problemática vinculada con este tipo movilidad en las representaciones sociales sobre las drogas fue en diciembre de 2010. En ese período, realizamos unas prácticas con la Asociación Proyecto Hogar, institución que intervenía (entre otros lugares) en la Glorieta de Embajadores.

En Madrid, la existencia de personas que quieren consumir droga, junto a la ubicación de los principales puntos de venta en la periferia de la ciudad, generó la necesidad de medios de transporte que llevasen a esta población a dichos espacios. Naciendo las "kundas" como respuesta. Tratándose de vehículos privados, en los cuales el conductor acerca a población consumidora a una zona de venta de droga. Allí los espera y regresa con ellos a Madrid. Cobrando un dinero a cada usuario, pudiendo aceptar droga como pago por sus servicios.

Si bien el origen del fenómeno "kunda" no puede ser ubicado en la actualidad, su proliferación si es coetánea. La dura crisis que sufrió este país hace no pocos años, propició que personas sin ingresos utilizaran este fenómeno como fuente de ingresos, dando respuesta a la necesidad de transporte que evidencia gran parte de la población drogodependiente, por carecer de él. Propiciando esta situación, un aumento de visibilización en las zonas de "carga y descarga" de pasajeros, aumentando la percepción de peligro.

Este hecho ocasionó un conflicto con gran ruido mediático. El cual decreció al aumentar la presencia policial en la zona, trasladando el espacio de "carga" de población drogodependiente, a otras calles aledañas.

\section{b) Zonas públicas de Madrid}

Otro conflicto ocasionado por visibilización de drogodependientes se produce en parques, zonas verdes y plazas municipales. Estos espacios, influidos por las pautas de ocio madrileñas y/o la cercanía de algún recurso asistencial, posibilitan la permanencia de un elevado número de población usuaria de drogas.

El consumo de alcohol y cannabis en estos espacios (fuera de sus zonas de uso tolerado), junto al gran estigma que portan estas personas, ocasionan que estos espacios sean significados en términos de peligro. Aumentando le percepción subjetiva del riesgo. Articulando respuestas por parte del vecindario para recuperar estos espacios, buscando encubrir aquello que han identificado como negativo.

La llegada del frío y las lluvias, junto al aumento de presencia policial y la modificación del mobiliario de estas zonas, provocan que las concentraciones disminuyan. Reduciendo paralelamente la conflictividad y la sensación de peligro. 


\section{c) Crisis en la calle Calella.}

A finales de 2016, en la calle Calella del distrito Puente de Vallecas, estalló un conflicto entre vecinos, comerciantes e instituciones, por la implantación de un recurso dispensador de metadona. Cuyo establecimiento provocaría la llegada a la zona de múltiples individuos vinculados con las drogas.

Tras muchas quejas, caceroladas, presiones a la junta municipal, reuniones de vecinos, acrecentado por un tratamiento tremendista por parte de medios de comunicación. etc. el recurso modificó las horas de reparto.

Esto, lejos de acabar con el "problema", dispersó a los usuarios y el nivel de alarma que estos provocaron. Decreciendo paralelamente el interés del fenómeno para los medios de comunicación, quedando esta problemática como algo residual en la zona.

En la actualidad el dispositivo funciona a la perfección, escuchándose sólo quejas sobre el deterioro y suciedad de la zona, comunes al resto del barrio.

Este tipo de conflictos, se han sucedido durante la instalación de recursos similares en todo Madrid. Apareciendo en todos ellos, un proceso de instrumentalización del riesgo y de modificación temporal del imaginario social sobre las drogas similar, como respuesta negativa a su implantación. El cual, funcionando a modo de profecía auto-cumplida, se mantiene hasta que aquello que evoca el peligro desaparece.

\section{d) La epidemia de los narcopisos.}

En septiembre de 2017, los medios de comunicación empezaron a centrar gran parte de sus noticias, en la aparición en Vallecas de viviendas en las que se vendía y consumía droga: los llamados "Narcopisos".

En pocas semanas la percepción de peligro fue tal que respuestas vecinales al fenómeno como manifestaciones, juntas de residentes, solicitudes de ayuda al gobierno etc. generaron tanto revuelo, que el suceso trascendió como problemática de interés nacional.

El incremento de policía en las calles, cambios en los protocolos de intervención sobre la vivienda ocupada, el cierre del algunos espacios de compraventa y un cambio en la dinámica de acceso a ellos por parte de sus usuarios, provocaron la invisibilización del fenómeno y por ende, una bajada en la percepción de peligro.

Actualmente las anteriores medias han provocado la vuelta de las " $k$ undas" al centro de Madrid (ya que, cerrando el mercado de proximidad, provocas que los usuarios tengan que ir desplazarse a los puntos de venta de nuevo), mostrando la inoperancia de políticas interventivas basadas en la represión. 
El análisis de los cuatro fenómenos anteriores a través del fenómeno NIMBY, evidencia dinámicas similares ante la implementación de ciertas instalaciones o la aparición de actividades percibidas como peligrosas por parte de la población madrileña. Estos sucesos, al generar visibilización de población drogodependiente, articulan una modificación temporal de las representaciones sociales sobre las drogas, utilizando la vuelta de "la epidemia de la heroina" como justificación de ciertas actuaciones contrarias al posicionamiento moral sobre las drogas que tienen al inicio del suceso. Postura a la que volverán, cuando la situación evocada como peligrosa sea desdibujada a través de las decisiones tomadas desde una instrumentalización de su percepción subjetiva del riesgo.

Este proceso evidencia cómo la inseguridad es un fenómeno construido socialmente sobre un modelo interpretativo de las adicciones negativizado, similar al proceso de construcción del concepto “yonki”, que tan bien explican Pallarés (1996) y Massó (2014) ${ }^{23}$.

Teniendo que analizar este fenómeno para no caer en el error de articular intervenciones desmesuradas que solo oculten de nuevo la realidad, como sucede en los casos anteriormente relatados.

\section{Características de las zonas de conflicto con población drogodependiente en Madrid Capital.}

UNAD, Asociación Proyecto Hogar e Instituto de Adicciones (2008) ${ }^{24}$, muestra las similitudes encontradas entre las distintas zonas en las que intervienen. Estos espacios presentados, al ser similares a los comentados en el apartado anterior, funcionaran como referencia en este texto para mostrar las características similares de aquellas zonas en las que se suceden conflictos como los aquí analizados.

Sin olvidar que, en la mayoría de las áreas de mediación de la asociación Proyecto Hogar, realizamos gran parte de nuestro trabajo de campo. Complementando con este, las ideas que estas organizaciones plasman en el anterior texto.

Estas particularidades no sólo generan acumulación, visibilización y definición en términos de conflicto de población drogodependiente, sino que ponen en marcha mecanismos cuya intencionalidad es devolverlos al ostracismo.

a) Buena comunicación por transporte público. Las zonas de narcopisos, los

23. Massó, P. (2014). "Un aleph en el callejón del gato. Espacio, cuerpo y ritual: una antropología de los tratamientos de heroína para la adicción a opiáceos en la biopolítica de la reducción de daños". (Tesis doctoral). Tarragona. Universidad Rovira i Virgili.

24. UNAD, Asociación Proyecto Hogar e Instituto de Adicciones (2008). "Manual de buenas prácticas en mediación comunitaria en el ámbito de las drogodependencias”. Madrid. Ayuntamiento de Madrid. 
dispositivos asistenciales, plazas, parques, espacios de salidas de "kundas", etc., suelen ser accesibles por este medio. Como muchos de los individuos que permanecen en ellas no cuentan con transporte propio, la existencia de este tipo de comunicación resulta crucial para la elección de un espacio respecto a otro.

b) Cercanía a lugares relacionados con las adicciones. Muchas de estas zonas están cerca de espacios vinculados a la droga. Lo que comenzó con el rechazo a dispositivos asistenciales, se extrapoló a todo aquello conectado real o simbólicamente con ella. Con la negativización y el aumento de la percepción del riesgo que esto supone.

c) Atractivo zonal. Estos territorios cuentan con infraestructuras funcionales, que motivan la permanencia de esta población. Los agentes sociales que establecen estos espacios como parte central de sus conductas, disponen de bastante tiempo libre. De ahí que lugares soleados, refugiados del viento, semi-ocultos, con bancos y mesas, cercanos a tiendas de alimentación, etc. decanten su elección con respecto a otros que no disponen de dichos elementos.

d) Deterioro. Las altas concentraciones de esta población provocan que, al ser identificados como peligrosos, asuman parte de los problemas acontecidos en la zona. El deterioro del mobiliario urbano, es una de las características de gran parte de las zonas verdes de Madrid. Situación ocasionada por el mal uso de la población en general y la falta de mantenimiento institucional. La visibilización de este colectivo, hace que asuma la autoría de estos desperfectos, a pesar de no ser los únicos responsables.

e) El referente de la "epidemia de heroína". Las zonas donde se generan estos conflictos, caracterizadas por haber tenido una relación pretérita con las adicciones, son significadas desde esta idea. Este pasado, reconstruido y/o magnificado en el discurso vecinal con una intencionalidad clara, sustenta algunas de las acciones llevadas a cabo en la actualidad, tengan o no que ver con las adicciones.

f) Persistencia asociacional. Estos espacios presentan asociaciones generadas durante la creación del barrio. Las cuales, asumieron responsabilidades que el estado no cubría, manteniéndose actualmente en estado latente para intervenir en cualquier situación de conflicto actual.

g) Proyectos de vida significados en negativo. Estos espacios, conformados por inmigración interna en el pasado y externa en la actualidad. En los que el precio de la vivienda es el menor de la capital. Con la mayor tasa de paro y la menor renta per cápita de toda la capital. Vinculados con las adicciones, etc. Provoca que los vecinos, influidos por la gran cantidad de hechos sociales estigmatizados con los que convive, definan su trayectoria vital desde lo negativo. Responsabilizando de esto a realidades negativizadas, en vez de reflexionar sobre su propio papel y el del estado, en la situación que viven. 


\section{Tipología de los agentes sociales que conviven en estas zonas.}

Mostradas las características comunes de las zonas donde se genera conflicto con población drogodependiente en Madrid, consideramos necesario añadir una tipología de actores que interaccionan en ellas. Facilitando con ello la comprensión del fenómeno analizado.

Siendo la relación mantenida con las drogas, la principal variable que caracteriza a los agentes sociales que interaccionan en estos espacios.

\section{- Población drogodependiente.}

Lejos de la definición de dependencia clásica de Kramer y Cameron (1975) ${ }^{25}$, sustentada en el triángulo de Lewin $(1970)^{26}$, abogamos por un concepto más cercano a la realidad para definir este hecho social.

"Cuando una persona consume un tipo de droga a diario, ésta ocupa un papel central en su vida, habrá desarrollado dependencia; mantendrá con la sustancia una relación de dependencia, distinguiéndose por esto de otros tipos”. Pallarés (1996).

Este autor, en la obra anteriormente citada, afirma que "de todos los consumidores de drogas, sólo una parte muy pequeña desarrollará dependencia a la sustancia”. Entendiendo el proceso como un itinerario de posibilidades, rompiendo con el concepto de Fernández y Sierra $(2008)^{27}$, de "escalada de la adicción".

En los contextos analizados, el conflicto es generado por la permanencia de individuos que evidencian mantener (tanto por sus actos, como físicamente) una relación de dependencia con alguna sustancia o están en tratamiento por ella. Estos, significados desde el "problema de la droga", provocan percepción de peligro, la cual se incrementa según el número avistado. Situación que provoca la implementación consciente o no, del proceso al que ya nos hemos referido.

Según la cuantía de la estancia, los drogodependientes serán:

25. Kramer, J.y Cameron, D.C. (1975). “Manual on drug dependence”. Ginebra. World Health Organization.

26. Lewin, L. (1970): “Phantastica. Drogues Psychédéliques. Stuefiants, Narcotiques. Excitants et Hallucinogines”. Paris. Payot.

27. Fernández, R. y Sierra, M. (2008). "Análisis teórico sobre prevención de drogodependencias en el marco de la educación para la salud: factores de riesgo y de protección”. En Acciones e Investigaciones Sociales, 26, pp (161-187). Zaragoza. 


\section{○ "Permanentes".}

Son personas que persisten largos períodos en los espacios observados, regresando de forma habitual a ellos. Gran parte de estas se encuentran inmersas en un proceso terapéutico para controlar la adicción, perpetuándose en el espacio debido a la cercanía del centro de tratamiento o a su lugar de residencia principalmente.

Esta situación, normaliza su presencia para el vecindario. La cual no es identificada como problemática. Situación que varía, si su número excede el socialmente aceptado o viene acompañado de individuos no identificados con su misma categoría.

\section{○ “Efímeros".}

Estos agentes sociales, caracterizan su estancia en las zonas de conflicto por la brevedad. Los aledaños de centros de tratamiento, salidas de medios de transporte hacia puntos de venta, inmediaciones de narcopisos, etc. ejemplifican los espacios donde principalmente pueden ser localizados.

El objetivo que lleva a estos individuos a estos espacios, es resuelto con celeridad. Abandonándolos en un corto período de tiempo.

Muchos de los sujetos que llegan a estos lugares no son conocidos. De ahí que su presencia no esté normalizada como en el caso anterior, provocando un aumento en la percepción del riesgo.

Ninguna de las zonas analizadas presentó únicamente a un tipo de los anteriores perfiles.

Aunque mayoritariamente plazas y parques aglutinan drogodependientes con permanencias largas, también observamos estancias cortas de consumidores venidos de otras partes de la ciudad. Al igual que zonas de paso como los espacios anexos a los centros de tratamiento, presentan drogodependientes en tránsito y otros que los utilizaban para socializar con sus iguales.

Esta situación genera contradicciones en las percepciones del resto de individuos con los que interactúan en las zonas. Ya que, si bien identifican a usuarios de drogas con estancias permanentes como "no peligrosos", la aparición de "desconocidos" y el aumento del número de cualquiera de las dos tipologías, incrementa la percepción de peligro. Poniendo en marcha el mecanismo de gestión del riesgo subjetivo, al que ya nos hemos referido. El cual se acentúa rápidamente, instrumentalizándose para justificar acciones que acaben con la situación que lo ha agravado. 


\section{- Población no drogodependiente.}

En los contextos analizados, las personas son definidas como drogodependientes al identificarse en ellos ciertos "marcadores físicos de la adicción". Contrario a como dice Wolff et al (2001) ${ }^{28}$ y siguiendo con las ideas explicadas en Fernández Piedra (2018) ${ }^{(15)}$, la falta de piezas dentales, de higiene, extremada delgadez, etc. son interpretadas socialmente como señales de adicción a las drogas.

Con lo que su carencia hace que seas posicionado significativamente en el polo contrario. Difícilmente vamos a saber si una persona mantiene una relación de dependencia con una sustancia a través de la observación o no. Con lo que este proceso significativo, obedece más a una construcción simbólica sustentado en "el problema de la droga", que a la realidad.

De todas las posibilidades existentes dentro de la población analizada, otra de las categorías decisivas para la convivencia será la residencia. Sin olvidar que ninguna de las divisiones presentadas es pura, encontrándolas todas ellas mezcladas en la realidad.

\section{○ Residentes en la zona.}

\section{- Vecino.}

Este concepto define la relación que mantienen personas que viven cerca unas de otras. Articulando "parentesco ficticio" y generando vínculos de confianza e intereses similares, por el mero hecho de compartir un contexto de residencia similar.

Relación que a su vez estructura otro sistema de relaciones en oposición, con aquellos que no cumplen este requisito: no vecino.

En nuestros contextos de análisis, la población con mayor cantidad de años de residencia ha modificado esta acepción. Construyendo una nueva referente significativo sobre variables como la edad, nacionalidad y ser propietario de una vivienda. La cual, sustituyendo al anterior, articula un nuevo juego de representaciones y necesidades sobre las que generar el sistema de relaciones y confianza al que nos referíamos antes.

\section{- No vecino.}

En nuestro contexto de estudio, este es definido como aquel que no cumple con los requisitos de propiedad, nacionalidad y edad pautados por el grupo generador del

28. Wolff, K. Farrell, M. Marsden, J. Monteiro, M. G. Ali, R. Welch S y Strang, J. (2001). "Revisión de los indicadores biológicos de uso ilegal de drogas, consideraciones prácticas y utilidad clínica”. En Revista de Toxicomanías.28. pp (5-27). Barcelona. 
discurso hegemónico: el vecino con mayor cantidad de años de residencia. Respondiendo esta construcción más a un proceso de legitimización de modos de actuar por parte del vecino tradicional empoderado por su larga trayectoria de residencia, que a la realidad social.

Como concluimos en Fernández Piedra y Corral $(2018)^{29}$ esta dicotomía estructura un sistema de explicación binario, ante preguntas inefables sobre lo que sucede en el barrio. En la cual, los vecinos tradicionales responsabilizan de aquello negativo que sucede a aquellos diferentes a ellos. Posicionándose ellos como generadores y referentes de las representaciones sociales y tomas de decisiones correctas, sobre los hechos sociales totales al estilo de Mauss (1971), que suceden y se interrelacionan en el barrio.

\section{$\bigcirc$ Comerciantes.}

Este grupo lo forman quienes ejercen cualquier actividad comercial, en inmuebles ubicados en las zonas analizadas. Algunos de los individuos más reaccionarios, tanto a nivel discursivo como en sus acciones, pertenecen a este conjunto. Ya que su fuente de ingresos se resiente ante la visibilización de esos "otros" definidos como negativos. Responsabilizados socialmente de todo lo malo que sucede en el barrio.

\section{La instrumentalización de las drogas en contextos de conflicto poblacional en Madrid capital.}

A modo de discusión, planteamos cómo ciertos conflictos vinculados con las drogas y producidos en diversas zonas de Madrid capital, han sido "sofocados" institucionalmente sin el análisis ni la calma que necesitan.

En estos espacios, la normalización de las adicciones, la atenuación del estigma con el que cargan los consumidores y las posibilidades asistenciales existentes, invisibilizan tanto de las drogas como de sus consumidores. De ahí que el riesgo percibido sobre a las adicciones sea mucho menor que en otras partes de Madrid. Tolerando en ellos acciones y comportamientos que, en otros, serían duramente reprobados.

De igual manera, el "problema de la droga" influye con más fuerza en las representaciones manejadas en estos lugares, al compartir un referente mítico de sufrimiento y luchas contra estas sustancias.

De ahí que, ante situaciones escabrosas que conlleven visibilización de población drogodependiente, se desate un proceso temporal de modificación en el imaginario social respecto a las adicciones, a través del cual se legitiman acciones contrarias a

29. Mauss, M. (1971). “Sociología y Antropología”. Madrid. Tecnos. 
sus posiciones de tolerancia de partida. Siempre que sirvan para que el suceso que ha generado la amenaza, deje de verse como tal.

Aumento de la presencia policía, manifestaciones, patrullas vecinales, expulsión de los consumidores del barrio caceroladas, petición de cámaras de seguridad, endurecimiento de leyes, etc. Son algunas de las medidas tomadas bajo la presión del vecindario. Las cuales, canalizadas por el tejido asociativo, no han conseguito acabar con la situación de conflicto. Sólo la ocultaron de nuevo.

La vuelta de las “kundas", la existencia a día de hoy de narcopisos y los conflictos a la puerta de centros de tratamiento, sólo hacen que refutar la idea que acabamos de expresar. Así como evidenciar la necesidad de un cambio en las políticas interventivas y en la gestión del riesgo social percibido, para poder acabar realmente con estas problemáticas.

Con este motivo hemos utilizado el concepto NIMBY en nuestro análisis. Dado que refleja correctamente los sucesos acontecidos en nuestro campo de estudio. Este fenómeno, al definirse como la reacción de unos individuos que se organizan para enfrentarse a los riesgos que supone la instalación en su entorno de actividades o instalaciones percibidas como peligrosas sin oponerse a las mismas, ejemplifica a la perfección el proceso de modificación temporal de las representaciones sociales sobre las drogas, que hemos visto en las zonas analizadas. Ambos no desaprueban aquello que genera el conflicto, bien sea una antena de televisión o un grupo de drogodependientes. Siendo aquello que produce el rechazo, la cercanía a su domicilio de algo identificado como negativo.

Lo novedoso que presentamos, es la idea de la instrumentalización, consciente o no, de la percepción subjetiva del riesgo. Así como la gestión de este. Proceso que funciona a modo de profecía auto-cumplida, ya que una situación construida, evoca un nuevo comportamiento. El cual hace que la situación generada se tome como verdadera.

En cuanto a la gestión del riesgo percibido, los individuos que evidencian la amenaza manejan, la incertidumbre relativa a aquello que han significado como peligroso, a través del proceso de modificación temporal de las representaciones sociales sobre la droga, que antes hemos explicado. Este, basado en la identificación, análisis y evaluación del peligro, genera una serie de estrategias de superación, utilizando saber experiencial de manera similar a la comentada por Espluga (2006), respecto al riesgo percibido en la instalación de antenas de telefonía móvil pero en nuestro contexto de estudio.

Ejemplificándolo con el fenómeno de los narcopisos, los datos reales niegan la existencia de un problema tan importante como refieren los medios de comunicación. El cual sí es evidenciado como tal, por el número irreal de estos instaurado el imaginario del vecindario. La elevada prevalencia de estos, construida conscientemente o no, justifica 
las acciones posteriores. Acrecentando la percepción construida de amenaza ante la existencia de un número tan ingente de estos espacios. Pero como acabamos de explicar, esta situación incrementada socialmente, es aceptada con veracidad al servir para suavizar el suceso que la ha generado. Siendo una situación (por lo menos respecto al número y a la amenaza) construida socialmente.

Además, la negativización de aquello que no se corresponde con lo tradicional, responde metafóricamente a la necesidad de respuestas y soluciones institucionales, que no existen en una realidad social compleja como la presentada en los espacios de nuestro estudio y analizada en Fernández Piedra y Corral (2018). ${ }^{30}$

Esta polarización genera interpretaciones simplistas de realidades complicadas, originando explicaciones en términos de problema, de problemáticas complejas y multivariables como el paro, inmigración y, por supuesto, las adicciones.

Así que, concluyendo el texto que está leyendo, podemos decir que la solución a las problemáticas analizadas ha de sustentarse en análisis profundos de las realidades sobre las que han de implementarse las decisiones políticas.

Los casos sobre los que gira este artículo raramente encontrará remedio si las tomas de decisiones se amparan en resoluciones rápidas y sin reflexión. Sin embargo, a través de programas de mediación (los cuales sirvan para mantener el nivel de alerta bajo), informaciones realistas, haciendo partícipe a todo el vecindario de las decisiones, otorgando medias compensatorias a los individuos afectados por los sucesos, desde la educación y las conclusiones de investigaciones científicas bien planteadas, los problemas encontrarán una solución acertada, no transitoria.

30. Espluga Trenc, J.(2006). "Dimensiones sociales de los riesgos tecnológicos : el caso de las antenas de telefonía móvil”. En revista de sociología .82. pp (79-95). Barcelona. 


\section{BIBLIOGRAFÍA.}

1. Fernández Piedra, D. y Corral, G. (2018). "Situación de la problemática de las adicciones en el Puente de Vallecas". Referencia de Exp.180201702546. Madrid. Madrid Salud Instituto de Adicciones.

2. Megías, E., et al. (2000). "La percepción social de los problemas de drogas en España”. Madrid. FAD.

3. Romaní, O. (1999). “Las Drogas. Sueños y razones”. Barcelona. Ariel.

4. (2010). "Adicciones, drogodependencias y "problema de la droga" en España: la construcción de un problema social. Cuicuilco. 17. Pp (83-101).

5. Pallarés, J. (1996). "El placer del escorpión. Antropología de las heroína y los yonquis”. Lérida. Milenio.

6. Comas Arnau, D. (1989). “La construcción social de la imagen del drogodependiente: consecuencias de la prevención y la atención”. Madrid. Inserso.

7. Escohotado, A. (1986). "La creación del problema (1900-1929)". En "Revista española de investigaciones sociológicas", 34, pp (3-56). Madrid.

8. Gamella, J. (1997). "Heroína en España, 1977-1996. Balance de una crisis de drogas”. En Claves de Razón Práctica, 72, pp (20-30). Madrid.

9. Sepúlveda, M., Báez, F. Y Montenegro, M. (2008). "No en la puerta de mi casa. Implantación no conflictiva de dispositivos de drogodependencias". Barcelona. Grup Igia.

10. Martínez Oró, D y Pallarés Gómez, J. (2013). “Riesgos, Daños y Placeres: A modo de introducción de un manual para entender las drogas". En "De riesgos y placeres: Manual para entender las drogas". P.p (23-36).

11. Glaser, B. \& Strauss, A. (1967). "The discovery of grounded theory”. Chicago. Aldine Press.

12. Berger, P y Kellner, H. (1985). “La reinterpretación de la sociología”. Madrid. Espasa Calpe.

13. Rose, N., O`malley, P. y Valverde, M. (2006). “Governmentality”. Annual Review of Law \& Society, 2, pp (83-104). Barcelona. 
14. Santodomingo, J. (2009). "Historia de las adicciones y su abordaje en España”. En "Historia de las adicciones en la España contemporánea". pp (13-36). Madrid. Ministerio de Sanidad y Consumo.

15. Fernández Piedra, D. (2018). “Narcóticos Anónimos y Centros de Atención al Drogodependiente: un análisis antropológico de dos recursos asistenciales". (Tesis doctoral). Madrid. Universidad Complutense de Madrid.

16. Sepúlveda, M., Montenegro, M. \& Báez, F. (2007). "NO en la puerta de mi casa. Implantación no conflictiva de dispositivos de drogodependientes". Barcelona. Grup Igia. Colección Cuestiones emergentes.

17. Kandel, D.B. (1980). "Developmental stages in adolescent drug involvement". "Theories on drug abuse". Rockville,Maryland. National Institute on Drug Abuse.

18. Alberdi, J., de la Peña, A. e Ibarra, P. (2002). "Una Reflexión Sobre La Acción Colectiva Nimby: El Caso Hontza". En Inguruak, Revista de Sociología y Ciencia Política. 33, pp (59 - 79). Bilbao.

19. Nello, O. (2003). “¡Aquí, no!: Els conflictes territorials a Catalunya”. Barcelona. Ediciones Empuries.

20. Sepúlveda, M., F. Báez Y M. Montenegro. (2008). "No en la puerta de mi casa. Implantación no conflictiva de dispositivos de drogodependencias". Barcelona. Grupo Igia.

21. Mardones Arévalo, R. (2009). “ ¡No en mi patio trasero!: el caso de la comunidad ecológica de Peñalolén!” En Íconos. Revista de Ciencias Sociales. 34, pp. (139-149). Quito.

22. Martín-Crespo, M. (1996). "Por qué sí y por qué no en mi patio de atrás. Una revisión del concepto de síndrome "Nimby" (Not In My Back Yard) en torno al tema de la gestión de residuos radiactivos". En Política y Sociedad, 23, pp (147-152). Madrid.

23. Massó, P. (2014). "Un aleph en el callejón del gato. Espacio, cuerpo y ritual: una antropología de los tratamientos de heroína para la adicción a opiáceos en la biopolítica de la reducción de daños". (Tesis doctoral). Tarragona. Universidad Rovira i Virgili.

24. UNAD, Asociación Proyecto Hogar e Instituto de Adicciones (2008). "Manual de buenas prácticas en mediación comunitaria en el ámbito de las drogodependencias". Madrid. Ayuntamiento de Madrid. 
25. Kramer, J. y Cameron, D.C. (1975). "Manual on drug dependence”. Ginebra. World Health Organization.

26. Lewin, L. (1970): "Phantastica. Drogues Psychédéliques. Stuefiants, Narcotiques. Excitants et Hallucinogines”. Paris. Payot.

27. Fernández, R. y Sierra, M. (2008). “Análisis teórico sobre prevención de drogodependencias en el marco de la educación para la salud: factores de riesgo y de protección". En Acciones e Investigaciones Sociales, 26, pp (161-187). Zaragoza.

28. Wolff, K. Farrell, M. Marsden, J. Monteiro, M. G. Ali, R. Welch S y Strang, J. (2001). "Revisión de los indicadores biológicos de uso ilegal de drogas, consideraciones prácticas y utilidad clínica”. En Revista de Toxicomanías.28. pp (5-27). Barcelona.

29. Mauss, M. (1971). “Sociología y Antropología”. Madrid. Tecnos.

30. Espluga Trenc, J.(2006). "Dimensiones sociales de los riesgos tecnológicos : el caso de las antenas de telefonía móvil." En revista de sociología .82. pp (79-95). Barcelona. 